\title{
Spectral Aliasing: A Super Zoom for 2D- NMR Spectra. Principles and Applications
}

\author{
Gaétan Bayiha Ba Njock ${ }^{\mathrm{abc}}$, Dieudonné Emmanuel Pegnyembª, Trixie Ann Bartholomeusz ${ }^{\mathrm{b}}$, \\ Philippe Christen ${ }^{\mathrm{b}}$, Bruno Vitorge ${ }^{\mathrm{d}}$, Jean-Marc Nuzillarde, Rupali Shivapurkarc, \\ Mohamadali Foroozandehc, and Damien Jeannerat ${ }^{\star c}$
}

\begin{abstract}
The NMR methodology based on spectral aliasing developed at the University of Geneva is reviewed. Different approaches aimed at increasing the resolution in the indirect carbon dimension of $2 \mathrm{D}$ heteronuclear experiments are presented with their respective advantages. Applications to HSQC, HMBC and other 2D heteronuclear experiments to the study of natural products and synthesis intermediates are shown. HSQC-based experiments for diffusion measurements, kinetics studies and titrations experiments all take advantage of spectral aliasing to reduce the experimental time from unrealistically long acquisition times to overnight experiments. The roles of computational methods such as DFT/GIAO and Logic for Structure Determination (LSD) in structure determination are discussed.
\end{abstract}

Keywords: Heteronuclear 2D NMR · HSQC-based experiments · NMR titration · Spectral aliasing · Structure determination of natural products

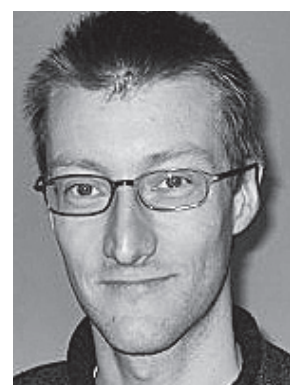

Damien Jeannerat was born in $\mathrm{Ge}$ neva (Switzerland) in 1970 and studied chemistry and biochemistry. In 1997, he obtained a $\mathrm{PhD}$ from the University of Lausanne after undertaking research at the National High Magnetic Field Laboratory in Tallahassee (Florida, USA) under the guidance of Professor Geoffrey Bodenhausen. He then joined the University of Geneva starting with a post-doc with Professor Ulrich Burger. He then became Maître Assistant

${ }^{*}$ Correspondence: Dr. D. Jeannerat ${ }^{c}$

Tel.: + 41223796084

Fax: + 41223793215

E-mail: damien.jeannerat@unige.ch

aDépartement de Chimie Organique

Faculté des Sciences

Université de Yaoundé I

P.O. Box 812, Yaoundé, Cameroun

'Section des Sciences pharmaceutiques

Université de Genève, Université de Lausanne

Quai E. Ansermet 30

$\mathrm{CH}-1211$ Genève 4

'Département de Chimie Organique

Université de Genève

Quai E. Ansermet 30

$\mathrm{CH}-1211$ Genève 4

dDépartement de Chimie associé au CNRS

Ecole Normale Supérieure

24 rue Lhomond

F-75231 Paris Cedex 05, France

eInstitut de Chimie Moléculaire de Reims

CNRS, UMR 6229, Université de Reims

Campus du Moulin de la Housse

F-51687 REIMS Cedex 2, France and since 2004 leads his research group as Maître d'Enseignement et de Recherche at the Department of Organic Chemistry. His interests are focused on the methodology developments in NMR of small molecules and their applications to chemistry and biology.

\section{Introduction}

The resolution in HSQC and other 2D heteronuclear spectra is usually not identical along the two axes. In the direct proton dimension, it is quite fine because it stems from the FID, but it is much coarser in the indirect carbon dimension to avoid exceedingly long experimental times. This asymmetry is especially pronounced in heteronuclear ${ }^{1} \mathrm{H}-{ }^{13} \mathrm{C}$ experiments such as HSQC and $\mathrm{HMBC}$ spectra where the resolution is typically close to $1 \mathrm{~Hz} / \mathrm{pt}$ in the proton dimension but often over $100 \mathrm{~Hz} / \mathrm{pt}$ along the ${ }^{13} \mathrm{C}$ chemical shift axis. If we compare NMR spectra with digital images, a typical HSQC is like the middle image in Fig. 1, where the finer features are blurred.

A higher resolution can be obtained by increasing the number of points in the carbon dimension until it reaches the limit of relaxation (image on the left of Fig. 1). The problem is that it also increases the experimental time by two orders of magnitude. This option not being viable, one has to turn to more practical alternatives. Spectral aliasing is a straightforward method that allows the increase of spectral resolution within the same total experimental time. It also permits the analysis of increasingly complex samples from the usual single molecule to mixtures of similar molecules, small libraries, etc. The com-

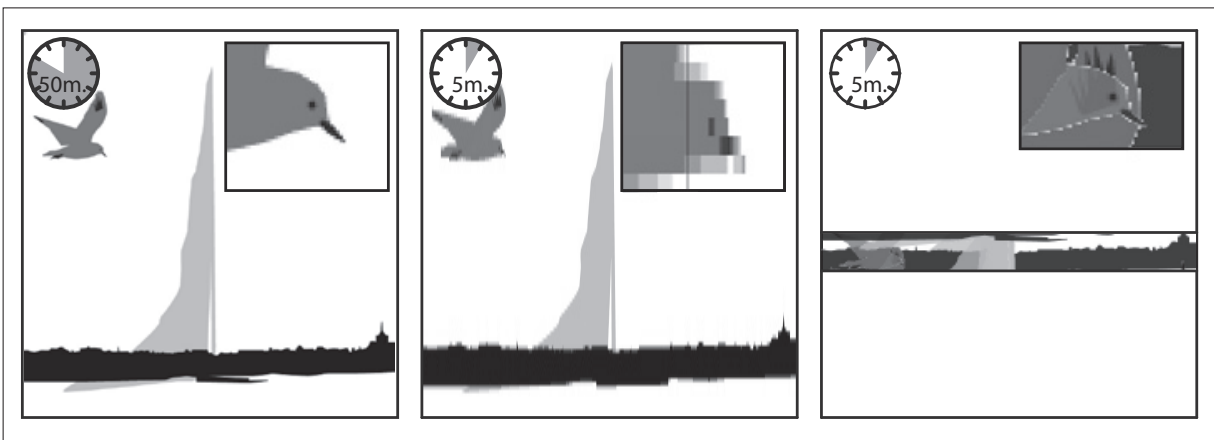

Fig. 1. Left: High-resolution image serving as comparison with spectra recorded with thousands of points in the indirect dimension and requiring long acquisition times. Middle: Effect of asymmetrical resolution as is typically obtained in 2D-NMR. Right: Effect of spectral aliasing where features are finely resolved but where slices overlap. The later two experiments require much shorter acquisition times and, after proper combination, provide the same information as the first. 
parison with digital images is illustrated on the right of Fig. 1 where the high resolution is obtained at the cost of superposition of slices. In this paper, we shall limit our presentation to spectral aliasing methods developed during the last few years in our laboratory. For a more complete review of the work in other groups and using other 'fast methods', the references in the cited papers herein may be consulted. Since we consider that applications should drive methodology developments, we will also present some selected applications illustrative of the type of problem one can solve using spectral aliasing in two- and threedimensional NMR.

Moving the center of a spectrum or changing its width so that the real frequencies are outside the spectral window causes signal aliasing or folding; as a result, the signals are located at deceptive positions on the actual spectral window. In NMR textbooks, this effect is often illustrated on 1D spectra but the digital filters of modern spectrometers eliminate these artifacts. One has to turn to $2 \mathrm{D}$ NMR to observe this effect. The virtue of reducing the spectral window of indirectly detected dimensions is the better resolution of signals into the spectral space as illustrated in Fig. 2. The acquisition times are similar to that of normal full-width spectra. In protein NMR, it is quite common to double the resolution by wrapping up large bands at the top and bottom of the ${ }^{15} \mathrm{~N}$ window, but we exploited this effect to a much larger scale in the carbon dimension of ${ }^{1} \mathrm{H}-{ }^{13} \mathrm{C}$ HSQC, HMBC, etc. We can therefore take advantage of the broad spectral range of carbons and the more favorable relaxation properties of small molecules to reach factors of improvements from 10 to $>100$.

\section{Spectral Aliasing in the Context of Structure Determination of Molecules of Natural Origin}

In a long-lasting collaboration with Dr. Philippe Christen, we studied numerous samples of natural origin. We shall illustrate some of our methodologies with a sample of podocarpusflavone B (putraflavone) found in the leaves of Campylospermum calanthum (Gilg.) Farron (Ochnaceae) originating from Cameroon (Fig. 3 ). This compound has previously been described in Podocarpus macrophylla D. Don (Podocarpaceae) from Japan, ${ }^{[1]}$ but this is the first time it has been isolated in an African plant.

The classical set of ${ }^{1} \mathrm{H}, \quad\left\{{ }^{1} \mathrm{H}\right\}-{ }^{13} \mathrm{C}$, DEPT-135, COSY, HSQC and HMBC experiments were recorded but structure determination was hindered by signal clustering due to the non-degenerated dimeric

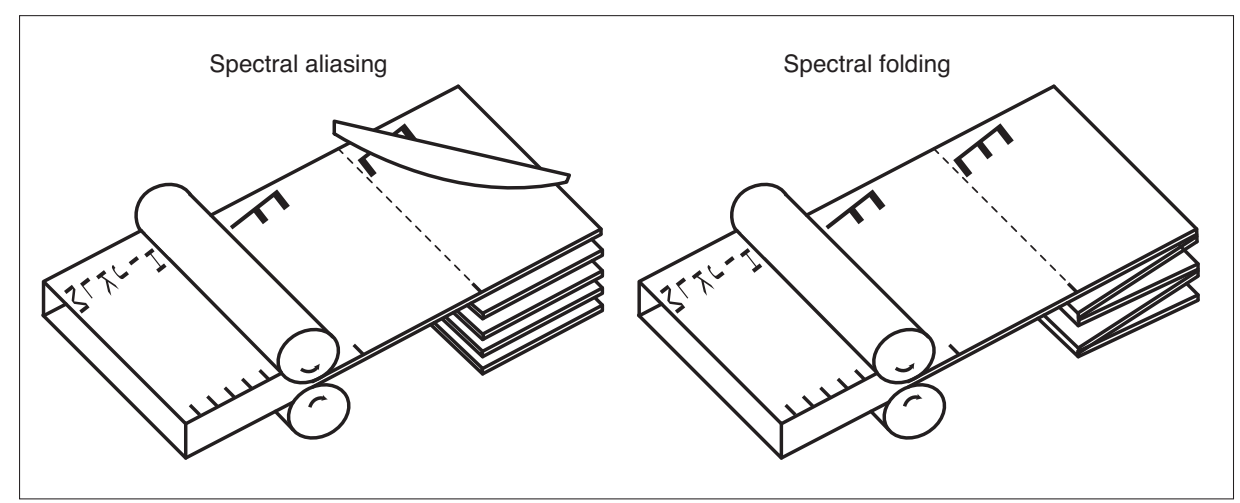

Fig. 2. Reducing the carbon spectral dimension of HSQC and HMBC spectra by a factor $n$ is like laminating a thick transparent spectrum and overlapping the $n$ slices. Signals that are too close to be resolved in the thick (normal full-width spectrum) are $n$ times better spread out in the thin spectral slices, but they may overlap with signals of other slices. In most cases, the quadrature detection causes spectra to be aliased (left) but TPPI causes signal folding (right).

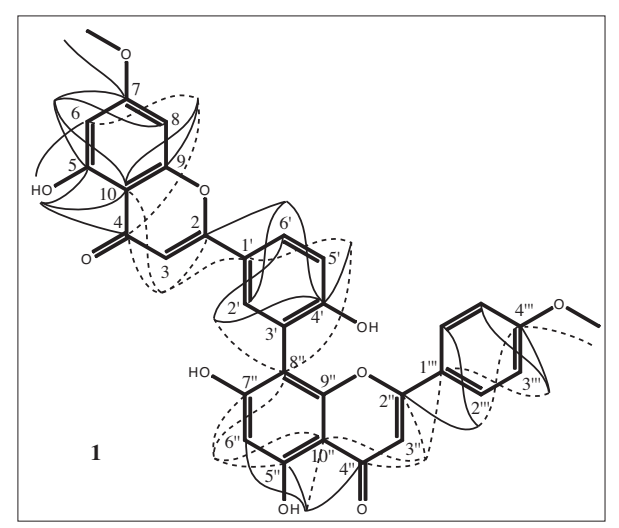

Fig. 3. Structure of podocarpusflavone B. The lines correspond to the HMBC correlations observed in the 10-ppm spectra. Note that only 22 of the 43 correlations were unambiguously assigned using the normal full-width HMBC spectrum and shown using continuous lines.

nature of the compound, causing serious assignment problems. The enlargement of the HMBC spectrum shown in Fig. 4 illustrates the difficulties.

\subsection{Assistance of Logic for Structure Determination (LSD)}

When the number of ambiguities due to signal overlap in HMBC spectra is overwhelming one can turn to computers to explore all the possible structures. Logic for Structure Determination (LSD) has been developed for this purpose by Nuzillard et al. ${ }^{[2]}$ The program essentially generates all the chemical structures compatible with the NMR connectivities coded in an input file. When the signals in 2D spectra are ambiguous, one simply provides the program with the list of all possible protons and carbons. When using data stemming from the normal full-width HMBC spectrum of $\mathbf{1}$, LSD proposed thousands of compatible structures. This situation can be improved by taking ad- vantage of the ability of the program to include user-defined structural information to limit the number of possible structures. In this study we suspected a flavonoid because of the yellowish color and the UV spectrum. After limiting our search to flavonoids as candidate structures, the situation improved dramatically since only eleven structures were proposed by LSD. Only five were compatible with the reasonable biosynthetic process imposing the oxygens to be located on carbons 5, 7 and 9 since they originated from a tri-acetate derivative. ${ }^{[3]}$ This illustrates the power of LSD to simplify problems when some structural information can be included or when heteroaromatic compounds with a large proportion of silent quaternary carbons are present. However LSD cannot solve severe overlap problems when nothing is known about the sample. A safer solution is therefore to resolve the signals by recording an aliased HMBC spectrum.

\subsection{Spectral Aliasing using 10-ppm ${ }^{13} \mathrm{C}$ Spectral Windows}

Setting the carbon spectral window of HSQC spectra to $10 \mathrm{ppm}$ is all that needs to be changed to a standard experiment to obtain a 15-20 fold resolution improvement. ${ }^{[4]}$ For HMBC experiments one may consider using pulse sequences tailored for long $\mathrm{t}_{1}$-evolution times. ${ }^{[4,5]}$ But, in both cases, the experiments are quite convenient and can be easily added to the set of standard NMR experiments. An example of comparison of a full and aliased spectra is shown in Fig. 4. In the classical spectrum, signals are ambiguous while the 10-ppm spectrum resolves all but one overlap! In the full spectrum, the last figures of the chemical shifts are unknown because of the width of signals in the carbon dimension. In the 10-ppm spectrum, one or two more figures can directly be read on the right of the period but the first two figures 


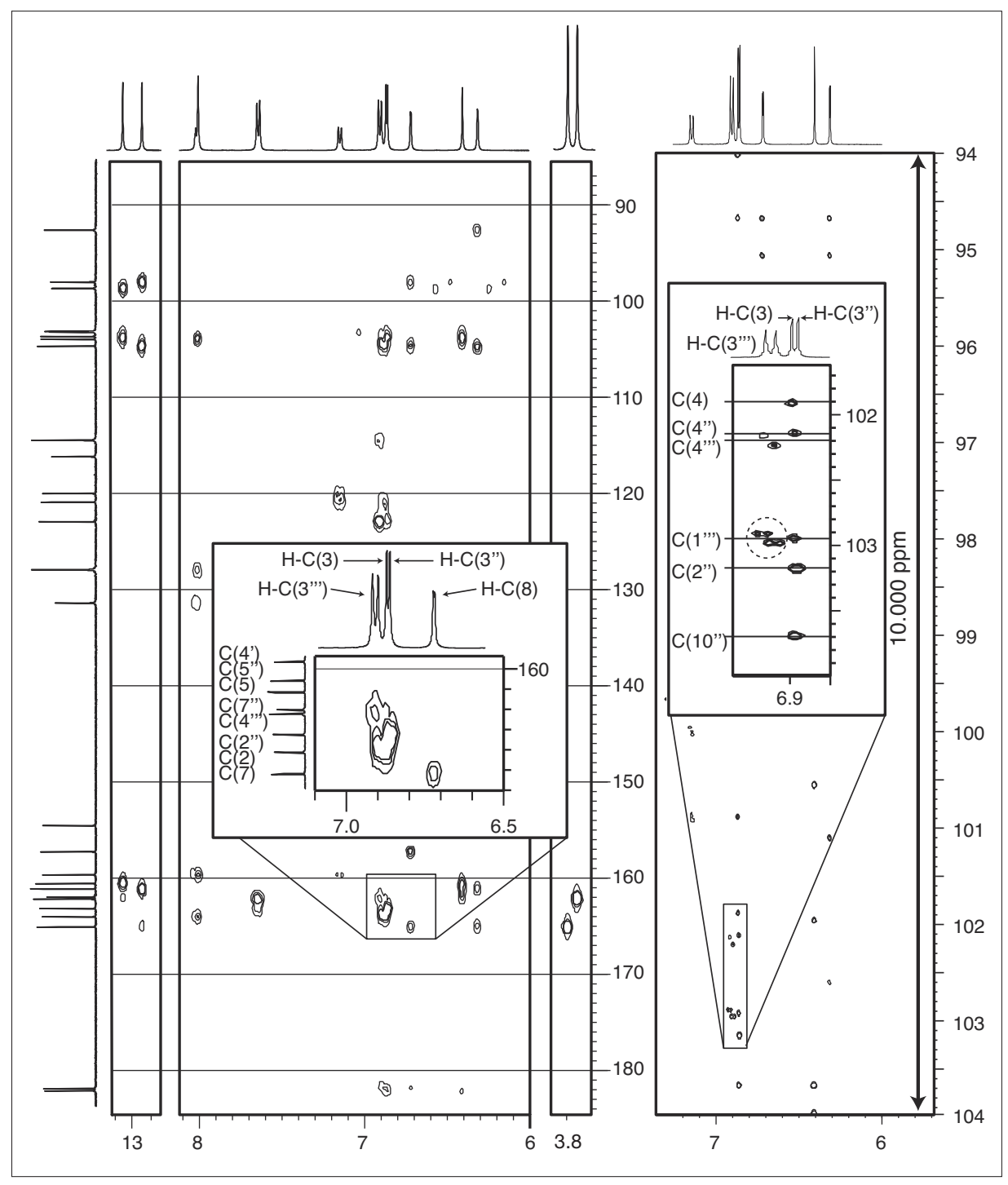

Fig. 4. Comparison of the HMBC spectra recorded using full and $10 \mathrm{ppm}$ spectral windows. Left: Normal spectrum recorded using $220 \mathrm{ppm}$. The chemical shifts are observed with $+/-0.2 \mathrm{ppm}$ errors. Right: $10-\mathrm{ppm}$ spectrum where the $+/-n^{\star} 10 \mathrm{ppm}$ ambiguities in the carbon chemical shift are solved using the full spectrum. The error on the chemical is $+/-0.01$. Dotted circle: The high resolution reveals the coupling structure of the signal correlating $\mathrm{H}-\mathrm{C}(3$ '”) with $\mathrm{C}(1$ '”).

on the left are unknown because of slice overlap. The combination provides the full and precise carbon chemical shifts. The structure determination therefore becomes straightforward with the 10-ppm spectrum. One could consider LSD to be of little help in this case, but we should see that it can actually facilitate structure determination and, more importantly, exclude other structures.

When provided with the correlations originating from the nearly unambiguous 10-ppm HMBC, LSD requires a fraction of a second to propose 36 structures. This set of solution mostly reflects the difficulty to position C(9") because no protons are close enough to this carbon to show signals in the HMBC. But a first advantage of LSD is that it quickly provides the definite fragments of the molecule in the form of the parts common to all proposed structures. have been seriously considered as a candidate before rejection. As a general rule for structure determination, and especially before publishing the structure of new compounds, one should in fact systematically use $\mathrm{LSD}^{[2]}$ to verify either the uniqueness of a structure or generate the exhaustive list of NMR-compatible alternatives. As an additional confirmation and anticipating the possible skepticism of a reviewer concerning the argument based on biosynthesis we turned to computation methods based on Density Functional Theory (DFT) and Gauge-Including Atomic Orbital (GIAO) and other carbon chemical shift calculations to test the two candidate structures.

\subsection{DFT/GIAO Methods as Complementary Information to Experimental NMR Data}

Nowadays existing software allows chemists to easily optimize the geometry of molecules and calculate the NMR shieldings of molecules with up to 30 carbons in a couple of days' calculation and a few hours of preparation and data analysis. We believe these techniques to be mature and ready for use by non-specialists as tools to complement NMR experimental data. We used such techniques to make a quick study of the two candidate structures generated by LSD. We also compared the experimental ${ }^{13} \mathrm{C}$ chemical shifts with values determined using the NMRShiftDB ${ }^{[6]}$ database and estimates from ChemBioDraw. Unsurprisingly the experimental spectrum matches the calculated ones for Fig. 3 much better than for the alternative structure. In this case the use of DFT/ GIAO methods may be seen as overkill because simpler calculations allowed us to reach the same conclusion, but they remain the only reliable approach for more subtle problems. In a collaboration with the group of Prof. Erick Carreira from ETH Zürich, we combined molecular dynamic, geometry optimization and chemical shielding calculations to determine the structures of slightly different slowly exchanging conformers. ${ }^{[7]}$

\section{Optimization of the Carbon Spectral Window}

Instead of using the convenient, but arbitrary spectral window of $10 \mathrm{ppm}$, we developed a computer program to determine the ideal spectral window to resolve all the signals with the minimal number of time increments. This approach, called COSA (Computer-Optimized Spectral Aliasing) ${ }^{[8]}$ consisted in taking a list of carbon chemical shifts and calculating the acquisition conditions to insure the maximal possible resolution whilst respecting a user-defined condition on the maximal $\mathrm{t}_{1}$ evolution time. 
This method is interesting for non-routine problems when one can return to the spectrometer for a second round of experiments tailored to fix the problem of a given sample. The optimization of the spectral window reduces the number of time increments and determines exactly how many are required to resolve all the peaks.

\subsection{Optimization Based on ${ }^{13} \mathrm{C}$ Data}

In its most simple version, the optimization simply requires a 'copy-and-paste' of a list of carbon chemical shifts to an application running on a web-server. When compared to 10-ppm spectra, a slight complication comes from the difficulty to interpret the chemical shift scale. In order to determine the real chemical shifts, it requires the addition of a special drawing layout designating the position of each carbon of the peak-list in the aliased dimension (see Fig. 5). This tool is available for a couple of NMR drawing programs and can be very easily adapted to others. The advantage of optimized spectra is that they insure the absence of overlap which can occur accidentally in 10-ppm spectra. An example of an optimized carbon dimension is shown in Fig. 5 and illustrates how the number of increments can be reduced once signals are homogeneously distributed along the carbon dimension. The factor of reduction depends on the number of carbons and the severity of the clustering of signals but is typically between 20 and 100.[5,9] One of the typical applications consists in recording a finely tailored ADEQUATE experiment when the carbon backbones of natural products have to be confirmed. ${ }^{[10]}$

\subsection{Inclusion of HSQC Spectra to the Optimization Process}

Compared to an optimization based only on a list of carbon chemical shifts, the additional information contained in a normal HSQC spectrum allows further optimization by permitting the overlap in the carbon dimensions for signals known to have different proton chemical shifts. This increases the density of signals in the aliased spectra and therefore requires fewer time increments. This is especially useful with molecules containing more than 25 carbons. The current implementation of this approach requires running a program on the computer that controls the spectrometer. When applied in a two-step manner ${ }^{[8]}$ to a sample of cyclosporine, an undecapeptide containing 53 protonated carbons, the acquisition time of the HSQC resolving all the signals was reduced by a factor of 125 (Fig. 6).

The great advantages of optimized experiments are to insure minimal recording times and open the possibility to record series of 2D experiments that could not be recorded using standard methods

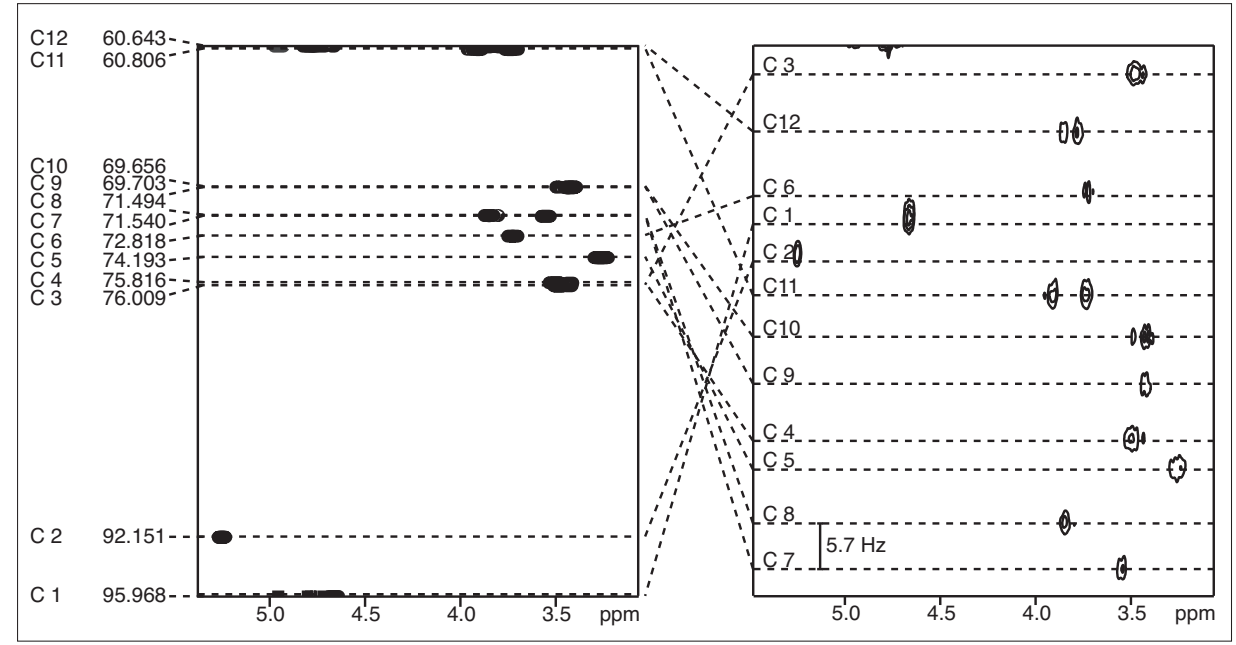

Fig. 5. Left: Normal HSQC spectrum of the two anomers of glucose dissolved in $\mathrm{D}_{2} \mathrm{O}$. In order to separate carbons 7 and 8, 3720 time increments would be necessary in the full 35 ppm spectrum. Right: Aliased spectrum recorded with the optimized spectral width of 0.554 ppm and 116 time increments. The reduction in acquisition time is $\times 32$.

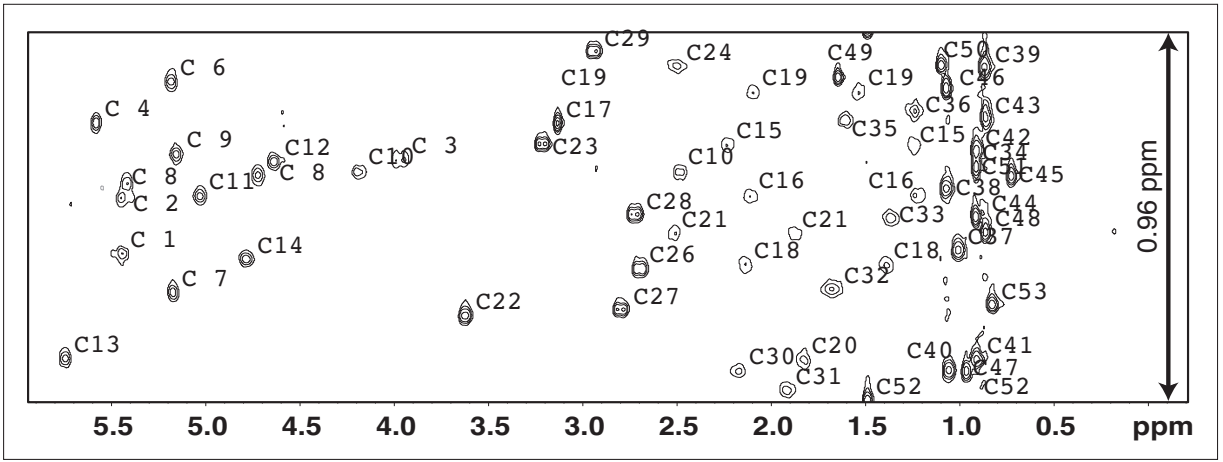

Fig. 6. Fully optimized HSQC of cyclosporine A, an undecapeptide containing 53 protonated carbons. The $0.96 \mathrm{ppm}$ spectral width was recorded with only 120 time increments. The acquisition conditions are ideal for relaxation measurements or any experiment where signal amplitude codes the measured quantity.

because of their extravagant needs of spectrometer time. Relaxation experiments are one example among the many possible applications we shall review in the next sections.

\section{Applications Based on Series of HSQC Spectra}

Series of $1 \mathrm{D}{ }^{1} \mathrm{H}$ NMR experiments are recorded in many different situations. In the textbook examples of relaxation time measurements, fitting the signal amplitudes as a function of a variable delay to the exponential function allows the determination of $\mathrm{T}_{1}$ or $\mathrm{T}_{2}$. Self-diffusion rates are more and more popular for giving insights, for example, about the state of ligands in solution with a metal. These diffusion rates are obtained using pulse sequences varying the intensity of pulse-field gradients. Many different types of titration experiments aimed at the measurement of affinity constants, determination of $\mathrm{pKa}$, identification of the binding sites of Lanthanide Shift Reagents (LSR), etc. all require recording series of spectra. In these cases, signal overlap may cause serious difficulties if not making the experiment impossible. Overlap problems also limit the complexity of the samples that can be analyzed using $1 \mathrm{D}{ }^{1} \mathrm{H}$ spectra.

A logical solution to overlap in $1 \mathrm{D}{ }^{1} \mathrm{H}$ spectra consists in spreading signals in a second dimension. COSY experiments being unreliable in significantly separating signals, we have recourse to an indirect carbon dimension. Exploiting the high gyromagnetic ratio of protons, HMQC and HSQC experiments are especially sensitive and partially compensate for the low natural abundance of ${ }^{13} \mathrm{C}$. When considering the extreme resolution attainable using spectral aliasing, the HSQC sequence is advantageous because signals do not show the elongation observed in HMQC spectra.

The acquisition parameters of the experiment are also important. In cases where overlap is a problem in the proton dimension, the standard full-width mode 
of acquisition is often insufficient to resolve signals in the carbon dimension or would require quite long acquisition times. This problem is especially critical in the perspective of recording series of 10-20 2D experiments. Spectral aliasing applied to HSQC experiments is therefore quite a powerful method to deal with complex samples such as mixtures of oligomers, complexes, inseparable natural products, etc. and limit the total acquisition time of a series of 2D spectra to a few hours instead of days.

The experiments based on series of HSQC spectra can be separated into two categories. When signals are expected to shift within the series of experiments, for example in titration experiments where the evolution of the chemical shift is the basis for the determination of the $\mathrm{pKa}$, it is not very useful to optimize aliasing and thus the arbitrary value for the carbon window of $10 \mathrm{ppm}$ (or less) is quite appropriate. The number of increments has to be large enough to provide the best possible resolution and insure empty space about each signal so that crossing trajectories are not too numerous. When the peaks are not expected to move, optimization of the spectral window is more advantageous except when the signal-to-noise ratio makes it useless to reduce the number of time increments to the minimum.

\subsection{Kinetic Measurements}

Kinetic measurements are often followed by ${ }^{1} \mathrm{H}$ NMR. The time needed to mix, lock, shim or reach a stable temperature makes it difficult to measure rate constants faster than $k=10^{-2} \mathrm{~s}^{-1}$ because nearly half of the reaction would be completed within the first minute. At the other end of the range accessible by NMR, rates above $10^{-5}$ are also difficult to determine because about only half of the reaction is complete after an overnight experiment. Luckily one can often influence these rates by adjusting the sample temperatures. Comfortable conditions consist in recording, say, 30 spectra within one hour of total experimental time. One option consists in recording single-scan experiments to neglect the variations in concentration during longer experiments. Alternatively, when the sensitivity requires recording signal continuously, one can take these variations into account when calculating the rate constants. In case of signal overlap due to the similarity of the molecules in the samples one can advantageously record 10-minute HSQC insuring good signal resolution using severe aliasing with windows of $1-5$ ppm. In such a case comfortable conditions for a full kinetic experiment are 4-6 hours or overnight experiments. Note that in the cases where only one pair of signals has to be resolved in the carbon dimension one can either use a small but arbitrary value for the carbon spectral window set according to signal-to-noise considerations or, when the difference in chemical shift $(\Delta \delta)$ between the two carbons is known, set the spectral windows to $\mathrm{SWa}=2 \Delta \delta$. In that case about ten time increments are sufficient to resolve the signals in the carbon dimension. In a collaboration with the group of Prof. Prins at the University of Padova (Italy) we investigated a small dynamic library of four hydrazine forming eight hydrazones and could follow the rate of exchange using a series of HSQC spectra. ${ }^{[11]}$ We designed a HSQC-based kinetic experiment where the unlucky overlap of just one pair of protons in the 1D spectra jeopardized a measurement involving a small library of similar molecules reacting with a template molecule. Because of signal-to-noise considerations, we optimized the carbon dimension so that more than one signal per molecule could be measured allowing us to complement our data with statistics on the precision and accuracy of the measurements.

\subsection{Diffusion Measurements}

Diffusion experiments often called DOSY (Diffusion-Ordered SpectroscopY) are usually recorded using series of $1 \mathrm{D}{ }^{1} \mathrm{H}$ sequences, but $2 \mathrm{D}$ variants have been developed. In principle one signal per molecule is all that is needed to measure its diffusion rate but, in complex cases, the difficulty lies in assigning an isolated signal to a component of the mixture. A carbon dimension is therefore quite useful in this context. The straightforward combination of the diffusion experiment with a HSQC is shown at the top of Fig. 7 where the two sequences are simply concatenated. The problem with such a sequence is that the pulse-field gradients available on standard equipment require diffusion times $(\Delta)$ lasting hundreds of milliseconds to discriminate molecules. When the second part of the sequence also takes hundreds of milliseconds to allow signals to be resolved, little signal is left when the acquisition of the FID finally starts. We therefore combined these two long delays into a Constant-Time HSQC to obtain a HSQC-iDOSY experiment ${ }^{[12]}$ which has been recently improved by Morris and coworkers. ${ }^{[13]}$

\subsection{Titration}

Titration experiments have many applications, some of which are listed in Table 1. In order to avoid having to use series of NMR tubes or impose manual additions, we developed a computer controlled system to make in-field additions between consecutive HSQC experiments (Fig. 8). The typical conditions for a titration experiment consists of adding the content of a $250 \mu \mathrm{l}$ syringe to a $500 \mu \mathrm{l}$ solution in 25 steps each taking typically 40 minutes (including sample mixing, equilibration, automatic shimming, and acquisition of a control ${ }^{1} \mathrm{H}-1 \mathrm{D}$ experiment). This allows an overnight full titration run. In order to avoid chemical shift changes due to sample dilution, the titrant usually contains the same concentration of the elements of the starting solutions. We used this setup to determine the pKa of a small library of acids in different solvents ${ }^{[14]}$ and currently work on more complicated networks of molecular interactions in future projects.

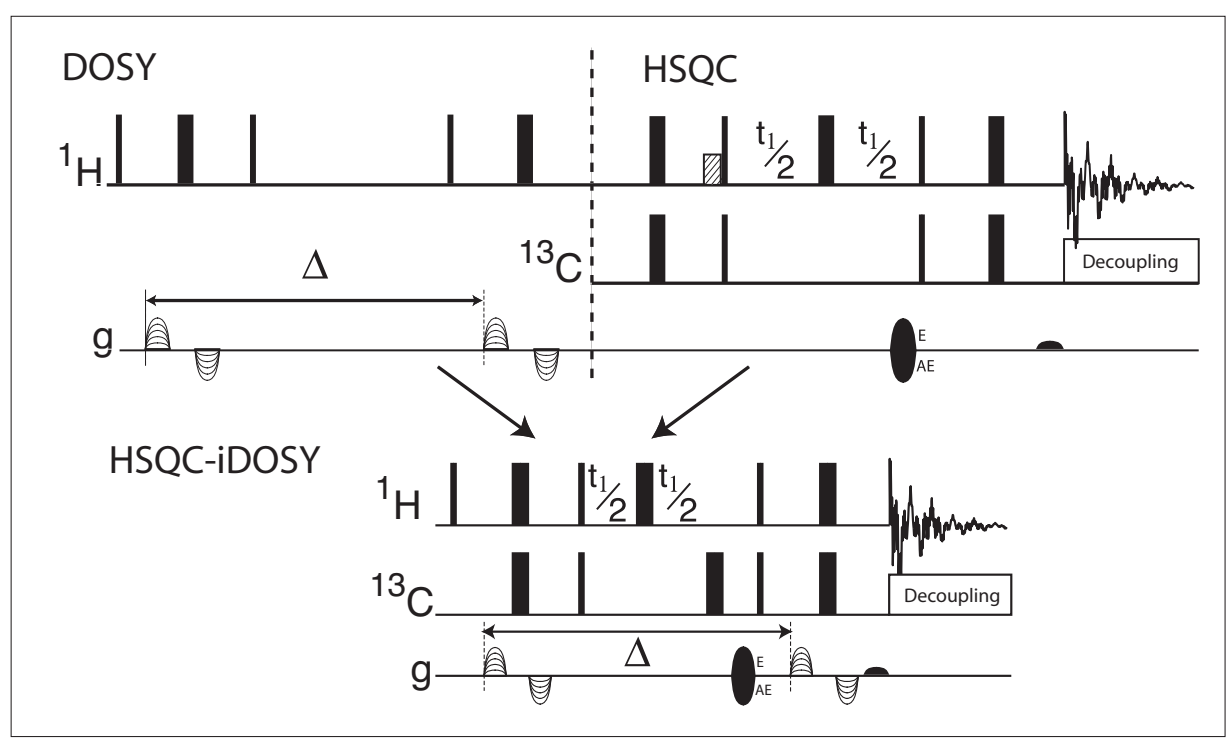

Fig. 7. Top: catenation of the dipolar version of a DOSY 1D pulse sequence with the HSQC. Bottom: 3D HSQC-iDOSY sequence combining the long diffusion and evolution delays between the diffusion gradients of a Constant-Time sequence. This sequence resolve most of the signals of a mixture of very similar carbohydrates. ${ }^{[12]}$ 


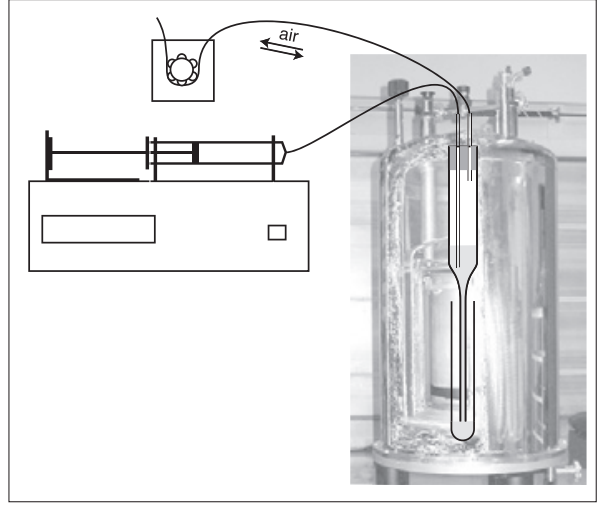

Fig. 8. Scheme of the automatic titration setup using a push-syringe for the addition and a peristaltic pump to mix the samples.

\section{Table 1.}

$\begin{array}{lll}\begin{array}{l}\text { Measu- } \\ \text { rement }\end{array} & \begin{array}{l}\text { Composition of } \\ \text { the library }\end{array} & \text { Titrant } \\ \Delta \mathrm{pKa} & \text { Bases } & \mathrm{H}^{+} \\ & \text {Acids } & \mathrm{HO}^{-} \\ \Delta \mathrm{K} & \text { Metals } & \text { Ligands } \\ & \text { Ligands } & \text { Metals } \\ & \begin{array}{l}\text { Carbo- } \\ \text { hydrates } \\ \text { Small } \\ \text { molecules } \\ \text { etc. }\end{array} & \text { Boronic acid } \\ & \text { Cyclodextrines } \\ & & \text { etc. }\end{array}$

\section{Applications using HSQC-based Experiments}

When COSY and NOESY spectra are too difficult to analyze because of extensive signal overlap, one can observe scalar and dipolar correlations between the source and the target protons in alternative experiments. HSQC-based experiments distributing these correlations according to the chemical shifts of the directly-bond carbons of the source of magnetization are less sensitive but are much more effective to resolve signals. These experiments are not new, but are more potent when combined with aliasing techniques to insure separation of all the carbons in the indirect dimension. These experiments turned out to be indispensable in the context of signal assignment and structure determination of a mixture of three similar molecules where 2D HSQC-TOCSY and HSQC-NOESY spectra provided the information usually obtained in COSY and NOESY (Fig. 9). ${ }^{[7]}$ Note that the resolution of signal in the carbon dimension being insured we chose the HSQC-TOCSY experiment instead of the COSY equivalent because relayed correlations are quite useful to resolve the few ambiguities due to proton overlap and do not cause overcrowding of the spectra, a
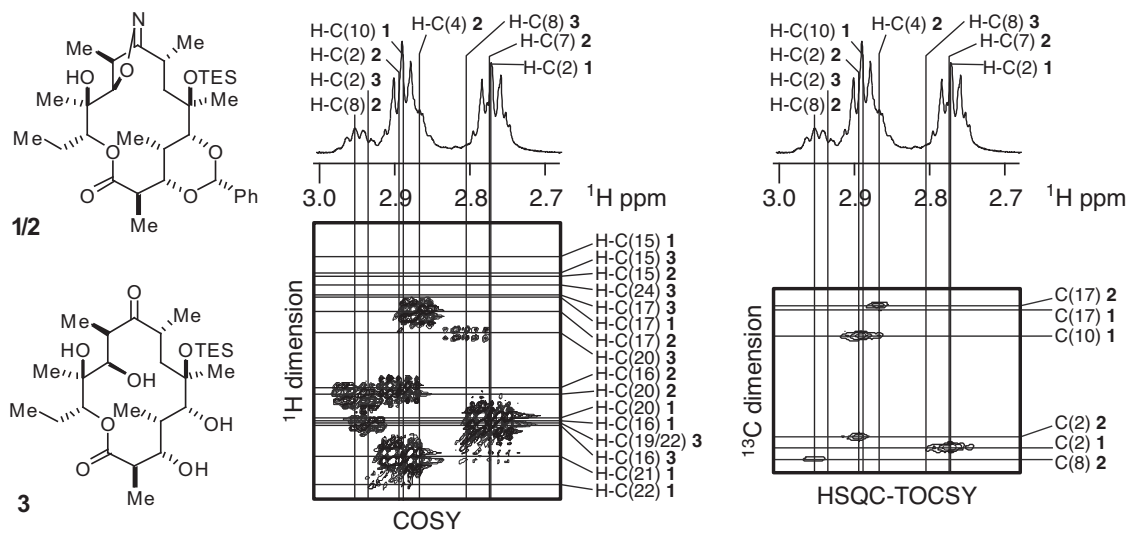

Fig. 9. Illustration of the ability of a carbon dimension to efficiently spread ${ }^{1} \mathrm{H}-{ }^{1} \mathrm{H}$ correlations of a mixture of two slowly exchanging conformers $\mathbf{1 / 2}$ and a decomposition product $\mathbf{3}$ that are too severely overlapping to allow for their assignment. Even a very finely-resolved DQF-DOSY spectrum (Middle) does not allow the identification of correlations, while individual carbons are easily assigned in the 2D HSQC-TOCSY (Right) recorded with an optimized carbon window. ${ }^{[7]}$

reason often justifying not recording homonuclear TOCSY experiments.

\section{Perspectives}

One of the future developments of our research will consist in the improvement of the methodology to facilitate the use of aliased spectra. We are developing novel combinations of spectra and innovative pulse sequences to avoid the need of a full-spectrum to determine the correct chemical shifts in 10-ppm spectra. Concerning future applications of our techniques, we can mention the measurement of Residual Dipolar Coupling, a very powerful method to determine molecular structures. These experiments provide rich and complementary information to those obtained using NOE and Karplus-based scalar coupling and should become very popular after simplification of sample preparation. These experiments usually involve the measurement of the ${ }^{1} \mathrm{~J}_{\mathrm{CH}}$ coupling constants in isotropic and anisotropic media. Spectral aliasing is interesting to observe these splittings in the proton or carbon dimensions but potentially even more interesting when using a third dedicated dimension. Such three-dimensional experiments, where the first two are the ${ }^{1} \mathrm{H}-{ }^{13} \mathrm{C}$ HSQC dimensions, open new opportunities for optimization. We also wish to move towards biological problems and contemplate developing methods at the interface between chemical biology, phytochemistry and metabolomics using ${ }^{13} \mathrm{C}$ and ${ }^{15} \mathrm{~N}$ enrichment when necessary. Even if it is not the most visible domain, we shall stay focused on liquid-state NMR of small molecules which still remains, and by far, the primary use of NMR spectrometers.

\section{Acknowledgements}

We thank André Pinto, Stéphane Grass and Patrick Romanens for technical support of the NMR spectrometers of the Department of Organic Chemistry. Funding comes from the Swiss SNF 2000021-11617 and the Département de l'Instruction Publique of the canton of Geneva. Jean-Marc Nuzillard acknowledges the support of the CNRS and Gaétan Bayiha Ba Njock the Swiss Department of Foreign Affairs for the International Scholarship.

Received: February 18, 2010

[1] H. Miura, T. Kihara, N. Kawano, Chem. Pharm. Bull. 1969, 17, 150 .

[2] J.-M. Nuzillard, Chin. J. Chem. 2003, 21, 1263; J.-M. Nuzillard, G. Massiot, Tetrahedron 1991, 47, 3655.

[3] B. A. Bohm, 'Introduction to Flavonoids', Harwood Academic Publishers, 1998.

[4] B. Vitorge, S. Bieri, M. Humam, P. Christen, K. Hostettmann, O. Munoz, S. Loss, D. Jeannerat, Chem. Commun. 2009, 950.

[5] D. Jeannerat, D. Ronan, Y. Baudry, A. Pinto, J.-P. Saulnier, S. Matile, Helv. Chim. Acta 2004, 87, 2190

[6] S. Kuhn, B. Egert, S. Neumann, C. Steinbeck, BMC Bioinformatics 2008, 9, 400.

[7] D. Muri, C. Corminboeuf, E. M. Carreira, D. Jeannerat, Magn. Reson. Chem. 2009, 47, 909.

[8] D. Jeannerat, J. Magn. Reson. 2007, 186, 112.

[9] Y. Baudry, D. Ronan, D. Jeannerat, S. Matile, Helv. Chim. Acta 2004, 87, 2181.

[10] L. H. Abdeljebbar, M. Humam, P. Christen, D. Jeannerat, B. Vitorge, S. Amzazi, A. Benjouad, K. Hostettmann, K. Bekkouche, Helv. Chim. Acta 2007, 90, 346.

[11] G. Gasparini, B. Vitorge, P. Scrimin, D. Jeannerat, L. Prins, J. Chem. Commun. 2008, 3034.

[12] B. Vitorge, D. Jeannerat, Anal. Chem. 2006, 78, 7601.

[13] A. S. McLachlan, J. J. Richards, A. R. Bilia, G. A. Morris, Magn. Reson. in Chem. 2009, 47, 1081.

[14] D. Jeannerat, R. Shivapurkar, in preparation, 2010. 